Pacific

Journal of

Mathematics

ON BIRATIONAL PROPERTIES OF SMOOTH CODIMENSION TWO DETERMINANTAL VARIETIES

IVAN PAN 


\title{
ON BIRATIONAL PROPERTIES OF SMOOTH CODIMENSION TWO DETERMINANTAL VARIETIES
}

\author{
IVAN PAN
}

\begin{abstract}
We show that a smooth arithmetically Cohen-Macaulay variety $X$, of codimension 2 in $\mathbb{P}^{n}$ if $3 \leq n \leq 5$ and general if $n>3$, admits a morphism onto a hypersurface of degree $(n+1)$ in $\mathbb{P}^{n-1}$ with, at worst, double points; moreover, this morphism comes from a (global) Cremona transformation which induces, by restriction to $X$, an isomorphism in codimension 1 . We deduce that two such varieties are birationally equivalent via a Cremona transformation if and only if they are isomorphic.
\end{abstract}

\section{Introduction}

Arithmetically Cohen-Macaulay (ACM for short) codimension 2 subschemes of $\mathbb{P}^{n}$ are geometric objects whose cohomologies satisfy very restrictive properties. In fact, the ideal sheaf of such a subscheme admits a determinantal resolution of length 2 whose Betti numbers determine a certain invariant, the so-called type (following the terminology of G. Ellingsrud [1975]) for the subscheme; in particular, most of their algebro-geometric properties are completely determined by this resolution. Ellingsrud showed that the ACM codimension 2 subschemes of $\mathbb{P}^{n}$ of a fixed type may be parametrized by an open, smooth and connected subset of a Hilbert scheme. Among these ACM subschemes, a still more special subfamily is that consisting of subschemes $X \subseteq \mathbb{P}^{n}$ whose ideal sheaf $\mathscr{F}_{X}$ has a determinantal minimal resolution of the form

$$
0 \longrightarrow \mathrm{O}_{\mathbb{P}^{n}}(-n-1)^{n} \stackrel{\mu}{\longrightarrow} \mathrm{O}_{\mathbb{P}^{n}}(-n)^{n+1} \longrightarrow \mathscr{F}_{X} \longrightarrow 0 .
$$

We will denote this family by $u_{n}$.

From geometers of the so-called Italian School of mathematicians, we know that such a general object is the base locus scheme of a Cremona transformation of $\mathbb{P}^{n}$ (see [Cremona 1871], [Cayley 1870], [Hudson 1927, Chapter XIV, Section 11] or Proposition 1); therefore it is not a complete intersection, following [Pan and Russo 2005, Proposition 2.1] (see also [Crauder and Katz 1991, Proposition 1]).

MSC2000: 13C40, 14E05, $14 \mathrm{E} 07$.

Keywords: Cremona transformation, determinantal variety, birational properties.

Partially supported by CNPq-Brazil, research grant 307833/2006-2. 
In this case, $X$ is smooth if and only if $n \in\{3,4,5\}$ [Ein and Shepherd-Barron 1989, Theorem 3.2] and thus constitutes a rare and therefore interesting object, as pointed out by Sample and Tyrrel [1970]. Another reason for exploring these kind of varieties is the special role they play in the Hartshorne conjecture on complete intersections: in fact, by a theorem due to Peskine and Szpiro [1974, Theorem 5.1], an ACM smooth codimension 2 subvariety of $\mathbb{P}^{n}$ is a complete intersection when $n>5$; hence, when $n>5$, varieties as in our setup yield examples which show that the smoothness hypothesis in Peskine and Szpiro's theorem is necessary.

In this work, we describe some birational properties of such smooth codimension 2 subvarieties. More precisely, first we show that for a smooth $X \in \mathcal{U}_{n}$ if $n=3,4,5$ and general $X$ for $n>3$, there exists a Cremona transformation $\phi_{X}: \mathbb{P}^{n}-->\mathbb{P}^{n}$ that induces, by restriction, a birational morphism $\eta: X \rightarrow Y$, where $Y$ is a hypersurface of degree $n+1$ in $\mathbb{P}^{n-1}$. Then we show that $\eta$ is an isomorphism in codimension 1, which is an isomorphism for $n=3,4$ and, at worst, a crepant resolution of a finite number of double points for $n=5$; from this we will conclude that a birational map $\varphi: X-->X$ is actually an automorphism; see Theorems 8 and 9. On the other hand, we show that two such smooth subvarieties of $\mathbb{P}^{n}$ are isomorphic if and only if they are birationally equivalent via a global birational map of $\mathbb{P}^{n}$; this is, essentially, the statement of Theorem 10.

The starting point for establishing our main results is the existence of the Cremona transformation $\phi_{X}$, whose construction is based on a very natural idea: We take general elements $V \in \mathbb{P} H^{0}\left(\mathscr{F}_{X}(n)\right)$ and $S \in \mathbb{P} H^{0}\left(\mathscr{F}_{X}(n+1)\right)$. The theory of linkage [Peskine and Szpiro 1974] shows that $X$ is linked to another $X^{\prime} \in \mathcal{U}_{n}$ by the complete intersection $V \cap S$; hence $V \in \mathbb{P H}^{0}\left(\mathscr{F}_{X^{\prime}}(n)\right)$. Thus $\phi_{X}$ is a Cremona transformation, of degree $n$, whose base locus scheme is $X^{\prime}$. We need to prove that the base locus scheme of $\phi_{X}^{-1}$ is smooth and also belongs to $u_{n}$. Then $\phi_{X}$ maps $V$ to a hyperplane, and one expects that it maps $S$ to an element of $\mathbb{P H}^{0}\left(\mathscr{F}_{X^{\prime}}(n+1)\right)$, from which it follows that $Y=\phi_{X}(V) \cap \phi_{X}(S)$.

\section{Some remarks on codimension 2 determinantal varieties}

Let $\mathbb{P}^{n}$ be the $n$-dimensional projective space over the field $\mathbb{C}$ of complex numbers. A Cremona transformation on $\mathbb{P}^{n}$ is a birational map $\phi: \mathbb{P}^{n}--\rightarrow \mathbb{P}^{n}$. We denote the base locus scheme of $\phi$ by $\operatorname{Base}(\phi)$. We can write

$$
\phi=\left(f_{0}: \cdots: f_{n}\right),
$$

where $f_{0}, \ldots, f_{n} \in \mathbb{C}\left[X_{0}, \ldots, X_{n}\right]$ are forms without common factors, of same degree, denoted by $\operatorname{deg} \phi$; the integer number $\operatorname{deg} \phi$ is the degree of $\phi$. If $Z \subseteq \mathbb{P}^{n}$ is an irreducible variety not contained in $\operatorname{Base}(\phi)$, we denote by $\widetilde{\phi}(Z)$ the closure of $\phi(Z \backslash \operatorname{Base}(\phi))$ and call it the strict transform of $Z$ by $\phi$. 
A linear system on $\mathbb{P}^{n}$ is the projective space associated to a vector space of forms on $\mathbb{C}^{n+1}$ of a certain fixed degree. We say a linear system $\Lambda$ is homaloidal if it has dimension $n$ and if the associated rational map

$$
\phi_{\Lambda}: \mathbb{P}^{n}-->\Lambda^{\vee}
$$

is birational. Clearly, every Cremona transformation is associated to a homaloidal linear system.

Let $X \subset \mathbb{P}^{n}$ be an ACM codimension 2 subscheme. There is a minimal resolution of the form

$$
0 \longrightarrow \sum_{j=1}^{n} \mathrm{O}_{\mathbb{P}^{n}}\left(-n_{j}\right) \stackrel{\mu}{\longrightarrow} \sum_{i=1}^{n+1} \mathrm{O}_{\mathbb{P}^{n}}\left(-d_{i}\right) \longrightarrow \mathscr{J}_{X} \longrightarrow 0,
$$

where $\mu$ corresponds to a matrix $M_{X}$ whose entries are (zero or) forms of degree $n_{j}-d_{i}>0$.

When $X$ is the base locus scheme of a Cremona transformation and $n \in\{3,4,5\}$, we know by [Pan and Russo 2005, Theorem 1.8] that $d_{i}=n$ and $n_{j}=n+1$ for all $i, j$. Conversely, suppose that we have a minimal resolution as above with $d_{i}=n$ and $n_{j}=n+1$, and $n$ arbitrary. The ideal sheaf $\mathscr{F}_{X}$ is generated by its global sections in degree $n$; more precisely [Peskine and Szpiro 1974, Section 3], the set of maximal minors $\Delta_{0}, \ldots, \Delta_{n}$ of $M_{X}$ is a minimal set of generators of it. Let $\nVdash i l b$ denote the Hilbert scheme attached to the Hilbert polynomial of $\mathcal{O}_{\mathbb{p} n} / \mathscr{E}_{X}$. The set of subschemes of $\mathbb{P}^{n}$ that have a resolution as in Equation (1) form an open and connected subset of Milb of dimension $n^{3}-n$ : see [Ellingsrud 1975, Theorems 1 and 2] or [Pan 1999, Cor. 2.1]. We denote this open set by $u_{n}$; we also write simply $X \in U_{n}$.

Therefore we now have a codimension 2 flat family $\mathscr{L} \subset \mathbb{P}_{\mathfrak{U}_{n}}^{n}=\mathbb{P}^{n} \times \mathcal{U}_{n}$ and a resolution

$$
0 \longrightarrow \mathcal{O}_{\mathbb{P}_{u_{n}}^{n}}(-n-1)^{n} \stackrel{\mu}{\longrightarrow} \mathcal{O}_{\mathbb{P}_{\mathfrak{U}_{n}}^{n}}(-n)^{n+1} \longrightarrow \mathscr{E}_{\mathscr{X}} \longrightarrow 0 .
$$

For the following result we adapt the deformation trick found in [Pan and Russo 2005, Theorem 1.8]. The same idea was used in [Gonzalez-Sprinberg and Pan 2006, Theorem 1] to show that the so-called multidegrees of a Cremona transformation $\phi$, whose base locus scheme is generically reduced and belongs to $u_{n}$, are exactly the binomial coefficients $\left(\begin{array}{l}n \\ k\end{array}\right)$ for $k=1, \ldots, n$; in particular, $\phi$ and its inverse $\phi^{-1}$ have degree $n$ in this case (we will use this in the proof below). We also observe that an easy (and known) exercise shows that if $\phi$ can be defined using the maximal minors of an $(n+1) \times n$ matrix of linear forms, perhaps not relatively prime, then its inverse map $\phi^{-1}$ has the same property.

Proposition 1. Let $\mathscr{B}_{n} \subset \mathcal{U}$ be the open set consisting of the subschemes $X \in \mathcal{U}_{n}$ whose associated linear system $\Lambda_{X}:=\mathbb{P}\left(\mathrm{H}^{0}\left(\mathscr{F}_{X}(n)\right)\right)$ defines a dominant rational 
map. Then there is a family of Cremona transformations with diagram

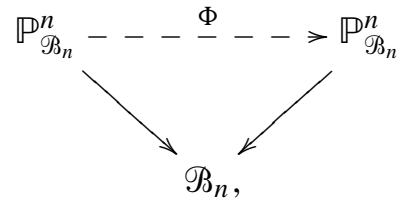

where we identify the dual of $\mathbb{P} \mathrm{H}^{0}\left(\mathscr{F}_{\mathscr{X}}(n)\right)$ with $\mathbb{P}_{\mathscr{P}_{n}}^{n}$ (that is, the map

$$
\Phi_{u}: \mathbb{P}^{n} \times\{u\}-->\mathbb{P}^{n} \times\{u\}
$$

is birational if $\left.u \in \mathscr{B}_{n}\right)$. Moreover, if $u \in \mathscr{B}_{n}$ corresponds to a generically reduced scheme, then $\operatorname{Base}\left(\Phi_{u}^{-1}\right) \in \mathscr{B}_{n}$.

Proof. The existence of a rational map $\Phi: \mathbb{P}_{\mathscr{Q}_{n}}^{n}--\rightarrow \mathbb{P}_{\mathscr{B}_{n}}^{n}$ is clear. For the first assertion it suffices then to prove that, for each $X \in \mathscr{B}_{n}$, the linear system $\Lambda_{X}$ is homaloidal.

Denote by $M_{0}$ the matrix of linear forms whose maximal minors define the Standard Cremona Transformation

$$
S_{n}=\left(\frac{1}{X_{0}}: \cdots: \frac{1}{X_{n}}\right) .
$$

The maximal minors of the parametric matrix $t M_{0}+(1-t) M_{X}$ for $t \in \mathbb{C}$ define a curve $T:=\left(t \mapsto u_{t}\right)$ when $t$ varies in $\mathbb{C}$ after eliminating, if necessary, a finite number of values $t_{1}, \ldots, t_{\ell}$.

Let $x, s_{n} \in \mathcal{U}_{n}$ be the points of $\mathcal{U}_{n}$ associated to $X$ and $\operatorname{Base}\left(S_{n}\right)$. Let $\mathscr{F}_{T} \subset$ $\mathbb{P}_{T}^{n}:=\mathbb{P}^{n} \times T$ be the flat family of the $u_{t}$ 's, parametrized by $t \in T$; notice that $x, s_{n}$ are regular points of $T$ because this curve can be realized as a line in the projective space whose homogeneous coordinates are the coefficients of the entries of the $(n+1) \times n$ matrix coming from the sequence in (3); see [Peskine and Szpiro 1974, Section 6]. Consider the blowing-up $\pi: \widetilde{\mathbb{P}_{T}^{n}} \rightarrow \mathbb{P}_{T}^{n}$ of $\mathbb{P}_{T}^{n}$ with center $\mathscr{F}_{T}$, and let $E$ be the exceptional divisor; denote the corresponding blowing-up in level $t \in T$ by $\pi_{t}: \widetilde{\mathbb{P}_{t}^{n}} \rightarrow \mathbb{P}_{t}^{n}$. Choose a general section $\mathscr{H} \in \pi^{*} \mathbb{O}_{\mathbb{P}_{T}^{n}}(n) \otimes \mathcal{O}_{\mathbb{P}_{T}^{n}}(-E)$.

The family $(\mathscr{H})_{t}$ is flat over $T$, and its members are the fibers of a dominant morphism; therefore the intersection number $\mathscr{H}^{n}$ is well defined. By the "conservation of number" (see [Fulton 1984, Corollary 10.2.1]) one has $(\mathscr{H})_{x}^{n}=(\mathscr{H})_{s_{n}}^{n}$. Now, the birationality of $S_{n}$ is equivalent to $(\mathscr{H})_{s_{n}}^{n}=1$, from which the birationality of $\Phi_{x}$ follows.

Finally, as we have remarked before, we know that $\operatorname{deg}\left(\Phi_{u}^{-1}\right)=\operatorname{deg}\left(\Phi_{u}\right)=n$; thus $\operatorname{Base}\left(\Phi^{-1}\right) \in \mathscr{B}_{n}$, completing the proof.

Corollary 2. There exists a birational map $u_{n}-->u_{n}$. 
Proof. The family of inverse maps $\Psi:=\Phi^{-1}$ defines a new family of Cremona transformations on $\mathbb{P}_{\mathscr{P}_{n}}^{n}$ such that $\Psi_{\mathrm{Base}\left(\Phi_{u}^{-1}\right)}=\Phi_{u}^{-1}$ for a generic $u \in \mathscr{B}_{n}$. The equation $\Phi \circ \Psi=\mathrm{Id}$ defines a relation on $\mathbb{P}_{\mathscr{P}_{n}}^{n} \times \mathbb{P}_{\mathscr{B}_{n}}^{n}$; this induces a generically one to one correspondence on $\mathscr{B}_{n} \times \mathscr{B}_{n}$.

Lemma 3. We have

$$
\operatorname{dim} \mathrm{H}^{0}\left(\mathscr{F}_{X}(k)\right)= \begin{cases}0 & \text { if } k<n, \\ n+1 & \text { if } k=n, \\ n^{2}+n+1 & \text { if } k=n+1 .\end{cases}
$$

Proof. By [Pan and Russo 2005, Proposition 1.2], we only need to prove case $k=n+1$. From Equation (2) we obtain an exact sequence

$$
0 \longrightarrow \mathrm{H}^{0}\left(\mathrm{O}^{n}\right) \longrightarrow \mathrm{H}^{0}\left(\mathcal{O}^{n+1}(1)\right) \longrightarrow \mathrm{H}^{0}\left(\mathscr{\Phi}_{X}(n+1)\right) \longrightarrow 0,
$$

from which the assertion follows.

Note that a generic $h \in \mathrm{H}^{0}\left(\mathscr{F}_{X}(n+1)\right)$ is irreducible. In fact $\mathrm{H}^{0}\left(\mathscr{F}_{X}(k)\right)=0$ for $k<n$, and the set of degree $n+1$ forms that are the product of a form of degree $n$ and a linear one is contained in a proper subvariety of $\mathbb{P H}^{0}\left(\Phi_{X}(n+1)\right)$.

Fix an irreducible $g \in \mathrm{H}^{0}\left(\mathscr{F}_{X}(n+1)\right)$, and denote by $S=V(g) \subseteq \mathbb{P}^{n}$ the subscheme defined by $g$. Take an irreducible element $V$ of the homaloidal linear system $\Lambda_{X}$. Then the scheme-theoretical equality $S \cap V=X \cup X^{\prime}$ holds, where $X^{\prime}$ is linked to $X$, and hence it is also a codimension 2 ACM scheme.

Remark 4. By Hartshorne's connectedness theorem, $X \cap X^{\prime}$ is of codimension 3 in $\mathbb{P}^{n}$.

Lemma 5. We have $X^{\prime} \in \mathcal{U}_{n}$.

Proof. As we know, $V=V(f)$ for an $f \in \mathrm{H}^{0}\left(\mathscr{F}_{X}(n)\right)$. Then

$$
g=\sum_{i} \lambda_{i} \Delta_{i} \quad \text { and } \quad f=\sum_{i} \mu_{i} \Delta_{i}
$$

where $\lambda_{0}, \ldots, \lambda_{n}$ are linear forms and $\mu_{0}, \ldots, \mu_{n} \in \mathbb{C}$.

The ideal sheaf $\mathscr{F}_{X^{\prime}}$ is generated by the maximal minors of a matrix $M^{\prime}$ obtained from $M_{X}$ by adding the $2 \times(n+1)$ matrix

$$
\left(\begin{array}{llll}
\lambda_{0} & \lambda_{1} & \cdots & \lambda_{n} \\
\mu_{0} & \mu_{1} & \cdots & \mu_{n}
\end{array}\right)
$$

The minors of $M^{\prime}$ are invariants, up to multiplication by a nonzero constant, by the natural (right) action of GL $(n+1)$. Hence one may suppose that $\mu_{0}=1$ and $\mu_{i}=0$ for $i=1, \ldots, n$. Thus we may replace $M^{\prime}$ by the matrix obtained from it by taking off the first column and the last row, and the proof is complete.

Proposition 6. Let $X \in \mathcal{U}_{n}$. The following statements hold: 
(a) $\operatorname{deg} X=n(n+1) / 2$.

(b) If $X^{\prime} \in U_{n}$ is linked to $X$, then it is done by way of a complete intersection of hypersurfaces of degree $n$ and $n+1$.

(c) The set of $X^{\prime} \in \mathcal{U}_{n}$ that are linked to $X$ is constructible and irreducible of dimension $n^{2}+n$.

(d) If $X$ is smooth and $X^{\prime}$ is general among the schemes as in (c), then $n \in\{3,4,5\}$ and $X^{\prime}$ is also smooth. In this case, a general hypersurface $V \in \Lambda_{X}$ (or $\left.S \in \mathbb{P} \mathrm{H}^{0}\left(\mathscr{F}_{X}(n+1)\right)\right)$ is smooth at points of codimension 2 , and points of codimension 3 have multiplicity $\leq 2 ; S$ is certainly singular if $n=5$.

Proof. Claim (a) follows directly from resolution (2). In particular, $\operatorname{deg}\left(X \cup X^{\prime}\right)=$ $n(n+1)$. Since $\mathrm{H}^{0}\left(\mathscr{\Phi}_{X}(k)\right)=\mathrm{H}^{0}\left(\mathscr{E}_{X^{\prime}}(k)\right)=0$ if $k<n$ (Lemma 3), this proves claim (b).

For the proof of (c), suppose $X \cup X^{\prime}=S \cap V=S^{\prime} \cap V^{\prime}$ holds scheme-theoretically, where $S, S^{\prime}$ have degree $n+1$ and $V, V^{\prime}$ have degree $n$; set $S=V(g), S^{\prime}=V\left(g^{\prime}\right)$, $V=V(f)$, and $V^{\prime}=V\left(f^{\prime}\right)$. Thus $X \cup X^{\prime} \subseteq V \cap V^{\prime}$, from which $V=V^{\prime}$ by (a). We deduce that $g^{\prime} \in(g, f)$, that is, there exist $\alpha \in \mathbb{C}$ and a linear form $\lambda$ such that $g^{\prime}=\alpha g+\lambda f$. Then the set

$$
\left\{S^{\prime} \in \mathbb{P H}^{0}\left(\mathscr{F}_{X}(n+1)\right): S^{\prime} \cap V=X \cup X^{\prime}\right\}
$$

is a projective space of dimension $n$.

Denote by $\mathbb{P}_{1}$ and $\mathbb{P}_{2}$ the projective spaces of forms on $(n+1)$ variables that vanish on $X$ and are of degree $n+1$ and $n$, respectively. The assertion (c) follows from a direct computation of dimensions involving the following commutative diagram and from using Lemma 3:

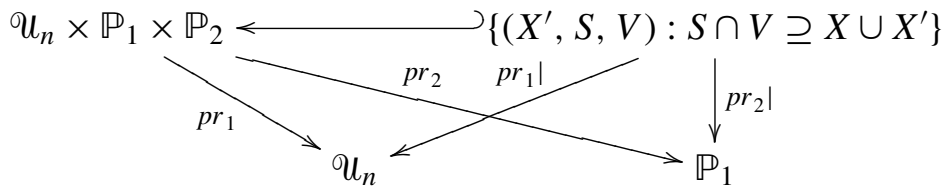

Here $p r_{1}$ and $p r_{2}$ denote the canonical projections, and $p r_{1} \mid$ and $p r_{2} \mid$ are their restrictions to the incidence variety.

Finally, let us consider now the situation of smooth $X \in U_{n}$. Since $\Lambda_{X}$ is homaloidal, we deduce $n=3,4$ or 5 according to [Ein and Shepherd-Barron 1989, Theorem 3.2]. Following [Peskine and Szpiro 1974, Proposition 4.1, parts (4) and (6)] we may choose $S$ and $V$ general enough so that $X^{\prime}, X \cap X^{\prime}, S \backslash\left(X \cap X^{\prime}\right)$ and $V \backslash\left(X \cap X^{\prime}\right)$ are all smooth.

Arguing as above, we may take $V, V^{\prime} \in \Lambda_{X}$ general enough so that $Y, X \cap Y$, $V^{\prime} \backslash(X \cap Y)$ and $V \backslash(X \cap Y)$ are all smooth, where $V \cap V^{\prime}=X \cup Y$. 
Fix a closed point $p \in X \cap Y$ (analogously for $X \cap X^{\prime}$ ) and take a general 2plane $\Pi$ passing through $p$. The traces of $V$ and $V^{\prime}$ on $\Pi$ define curves $C$ and $C^{\prime}$, respectively, passing through $p$. Since $\Pi$ intersects $X$ and $Y$ transversely, we conclude that $p$ is a point of length 2 in $\Pi \cap\left(X \cup X^{\prime}\right)$. Thus $p$ can not be a common singular point of $V$ and $V^{\prime}$ (respectively $V$ and $S$ ). In particular:

- $V$ does not have a singular point of codimension 2 since, by Bertini's theorem, such a point corresponds to an irreducible component of $X \cap Y$ and is also singular for $V^{\prime}$; the same argument works for $S$.

- A point of codimension 3 of $V$ has multiplicity $\leq 2$; it corresponds to a subvariety of codimension 3 of $V$ contained in $X \cap Y$.

In the case of $S$ that is a general determinantal variety, we need to show that singularities must appear in codimension 3 points.

Let $N=(n+1)^{2}-1$. Denote by $M_{k} \subset \mathbb{P}^{N}$ for $k \leq n+1$ the determinantal variety of matrices of rank at most $k$; here $M_{n+1}=\mathbb{P}^{N}$. It is well known that $M_{k}$ is the singular locus of $M_{k+1}$ and that it has codimension $(n-k+1)^{2}$ in $\mathbb{P}^{N}$; see for example [Arbarello et al. 1985, Chap. II]. On the other hand, the matrix defining $S$ induces an embedding $\mu: S \rightarrow M_{n}$. Therefore $\operatorname{Sing}(S) \supseteq \mu^{-1}\left(M_{n-1}\right)$. We conclude the proof by considering the case $n=5$.

Remark 7. (a) We do not know if singularities of $S$ must appear in codimension 3 , though the study of this kind of variety is a very classical subject.

(b) The indeterminancy of the linear system $\mathbb{P H}^{0}\left(\mathscr{F}_{X}(n+1)\right)$ is resolved by blowing up $\mathbb{P}^{n}$ along $X$ : indeed, if $\Lambda_{X}=\mathbb{P}\left(\left\langle f_{0}, \ldots, f_{n}\right\rangle\right)$, this holds for the linear subsystem

$$
\mathbb{P}\left(\left\langle x_{0} f_{0}, \ldots, x_{n} f_{0}, \ldots, x_{0} f_{n}, \ldots, x_{n} f_{n}\right\rangle\right) \subseteq \mathbb{P H}^{0}\left(\mathscr{F}_{X}(n+1)\right)
$$

because the homogeneous ideals $\left(x_{0} f_{0}, \ldots, x_{n} f_{0}, \ldots, x_{0} f_{n}, \ldots, x_{n} f_{n}\right)$ and $\left(f_{0}, \ldots, f_{n}\right)$ have the same saturation.

\section{The main results}

Definition 1. Let $X_{1}, X_{2} \subseteq \mathbb{P}^{n}$ be two subschemes. We say that the pairs ( $\mathbb{P}^{n}, X_{1}$ ) and $\left(\mathbb{P}^{n}, X_{2}\right)$ are birational equivalent if there exists a commutative diagram

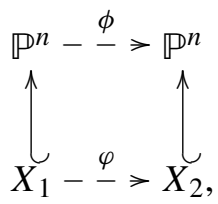

where $\phi: \mathbb{P}^{n}-\rightarrow \mathbb{P}^{n}$ and $\varphi: X_{1}--\rightarrow X_{2}$ are birational maps. 
We denote by $\operatorname{Bir}(X)$ the group of birational maps from $X$ to $X$, and by $\operatorname{Aut}(X)$ the subgroup of automorphisms of $X$.

Let $W$ be a normal projective variety. A Weil canonical divisor $K_{W}$ of $W$ is the closure of a canonical divisor of $W \backslash \operatorname{Sing}(W)$. Suppose there exists a positive $m \in \mathbb{Z}$ such that $m K_{W}$ is a Cartier divisor, and that for some desingularization $\pi: Z \rightarrow W$ there exists a divisor $F$, with $\pi_{*}(F)=0$, such that $m K_{Z} \sim \pi^{*}\left(m K_{W}\right)+F$. Under these hypotheses, we say that $W$ has canonical singularities if $F$ is effective; see [Debarre 2001, Section 7.2] or [Kollár and Mori 1998, Section 2.3]. When $F=0$ the desingularization is said to be crepant; see [Kollár and Mori 1998, Def. 6.22]. We now state and prove our main results.

Theorem 8. Given $n \in\{3,4,5\}$, let $X \in \mathcal{U}_{n}$ be a smooth codimension 2 subvariety; for $n>3$ we suppose in addition that $X$ is in general position. Then there exists a Cremona transformation $\phi: \mathbb{P}^{n}-->\mathbb{P}^{n}$ such that

(a) the restriction of $\phi$ to $X$ induces a birational morphism $\eta: X \rightarrow Y$, where $Y$ is a hyperplane section of a determinantal hypersurface of degree $n+1$;

(b) $\eta$ is an isomorphism in codimension 1, which is an isomorphism if $n=3,4$ and, at worst, a crepant resolution of a finite number of double points if $n=5$.

In the last case, over each double point there is a unique rational curve.

Theorem 9. Given $n \in\{3,4,5\}$, let $X \in \mathcal{U}_{n}$ be a smooth codimension 2 subvariety; for $n>3$ we suppose in addition that $X$ is in general position. Then $\operatorname{Aut}(X)=$ $\operatorname{Bir}(X)$.

Theorem 10. Given $n \in\{3,4,5\}$, let $X_{1}, X_{2} \in U_{n}$ be two smooth codimension 2 subvarieties; for $n>3$ we suppose in addition that $X_{1}$ and $X_{2}$ are in general position. The following statements are equivalent:

(a) $\left(\mathbb{P}^{n}, X_{1}\right)$ and $\left(\mathbb{P}^{n}, X_{2}\right)$ are birational equivalents.

(b) $X_{1}$ and $X_{2}$ are birational equivalents.

(c) $X_{1}$ and $X_{2}$ are isomorphic.

For the proof of Theorem 10 we need some technical lemmas; along the way we also prove Theorem 8 and then Theorem 9. First we explain our main argument.

Main construction. Fix $X \in \mathcal{U}_{n}$ smooth and general for $n \in\{3,4,5\}$. Take two general elements, $V$ in the homaloidal linear system $\Lambda_{X}$ and $S \in \mathbb{P H}^{0}\left(\mathscr{F}_{X}(n+1)\right)$. Then $S \cap V=X \cup X^{\prime}$, where $X^{\prime} \in \mathcal{U}_{n}$ is general as well; in particular it is smooth. Therefore there exists a Cremona transformation $\phi: \mathbb{P}^{n}-->\mathbb{P}^{n}$ for which $\operatorname{Base}(\phi)=X^{\prime}$ and $X^{\prime \prime}:=\operatorname{Base}\left(\phi^{-1}\right) \in \mathcal{U}_{n}$ is smooth.

Conversely, let $\phi: \mathbb{P}^{n}-->\mathbb{P}^{n}$ be a determinantal Cremona transformation of degree $n$ that is general in the sense that $\operatorname{Base}(\phi)$ is a general element in $\varkappa_{n}$; also 
suppose $\operatorname{Base}\left(\phi^{-1}\right) \in U_{n}$ is smooth. Hence we may link $\phi$ to elements $X \in \mathcal{U}_{n}$ in general position.

The strategy for proving Theorem 8 is to restrict to $X$ one of its linked Cremona transformations in order to transform it into a hyperplane section of a general determinantal hypersurface of degree $n+1$.

As we saw, $\Lambda_{X^{\prime}}$ is also homaloidal (Proposition 1); note $\phi: \mathbb{P}^{n}-->\mathbb{P}^{n}$ is a Cremona transformation with associated linear system $\Lambda_{X^{\prime}}$. Hence the strict transform of $V$ by $\phi$ is a hyperplane $H \subseteq \mathbb{P}^{n}$.

Lemma 11. Let $n \in\{3,4,5\}$. The map that associates the strict transform $\widetilde{\phi}(S)$ to a general $S \in \mathbb{P} H^{0}\left(\mathscr{F}_{X^{\prime}}(n+1)\right)$ induces an isomorphism

$$
\mathbb{P} \mathrm{H}^{0}\left(\mathscr{F}_{X^{\prime}}(n+1)\right) \simeq \mathbb{P} \mathrm{H}^{0}\left(\mathscr{F}_{X^{\prime \prime}}(n+1)\right) .
$$

Proof. Let $p: Z \rightarrow \mathbb{P}^{n}$ be the blowup of $\mathbb{P}^{n}$ along $X^{\prime}$; denote by $E$ its exceptional divisor. Letting $q$ be a birational morphism defined by the complete linear system $\left|p^{*} \mathcal{O}(n) \otimes \mathcal{O}(-E)\right|$, we have a commutative diagram

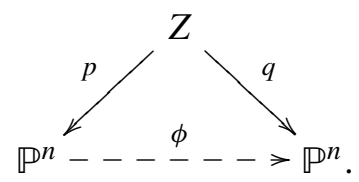

Since $X^{\prime \prime}=\left\{y \in \mathbb{P}^{n}: \operatorname{dim} q^{-1}(y) \geq 1\right\}$ is smooth, it follows from [Ein and ShepherdBarron 1989, Theorem 1.1 and Lemma 2.4] that $q$ is the blowup of $\mathbb{P}^{n}$ along $X^{\prime \prime}$ and $q(E)$ is an irreducible variety of degree $n^{2}-1$; this variety is the jacobian of $\phi^{-1}$, that is, the hypersurface defined by the jacobian determinant associated to the $(n+1)$ polynomials defining $\phi^{-1}$.

Let $S$ be a general hypersurface of degree $n+1$ containing $X$. It is singular at points that have codimension 2 in $X$; see Proposition 6(d). If $H \subseteq \mathbb{P}^{n}$ is a general hyperplane, then $p^{*} S$ is linearly equivalent to $(n+1) p^{*} H+E$, from which we conclude that $q_{*} p^{*} S$ is linearly equivalent to a hypersurface of degree $(n+1) n-$ $\left(n^{2}-1\right)=n+1$ containing the base locus scheme of $\phi^{-1}$.

Remark 12. We conclude that the strict transform of $X$ by $\phi$ is a hypersurface of $H=\mathbb{P}^{n-1}$ of degree $n+1$. We denote this variety by $Y=Y_{X} \subseteq \mathbb{P}^{n-1}$; observe that $\left(\mathbb{P}^{n}, X\right)$ is then birationally equivalent to $\left(\mathbb{P}^{n}, Y\right)$.

Proof of Theorem 8. The case $n=3$ is an easy consequence of Lemma 11 and Remark 12 because $Y$ is a quartic plane curve of geometric genus 3 (and therefore smooth). In what follows, we assume $n>3$ and keep all notations from Lemma 11 and its proof. 
The commutative diagram in Equation (5) gives also a resolution of indeterminancies of the inverse map $\phi^{-1}$ of $\phi$; let $F=q^{-1}\left(X^{\prime \prime}\right)$ denote the exceptional divisor of $q$. As we know, $q: Z \rightarrow \mathbb{P}^{n}$ is the blowup of the smooth variety $X^{\prime \prime}$.

Denote by $\tilde{X}, \widetilde{S} \subseteq Z$ the strict transforms by $p^{-1}$ of $X$ and $S$, respectively; we may suppose that $\widetilde{S}$ is smooth, since $p$ resolves the indeterminancy of the linear system $\mathbb{P} \mathrm{H}^{0}\left(\mathscr{F}_{X}(n+1)\right)$; see Remark 7(b).

Since $X \cap X^{\prime}$ has codimension 1 in $X$ (see Remark 4), $p$ induces an isomorphism from $\tilde{X}$ onto $X$; then it suffices to show that $q$ restricted to $\tilde{X}$ gives a birational morphism $\eta: \widetilde{X} \rightarrow Y$ satisfying the required properties.

When we restrict $q$ to $\widetilde{X} \backslash(F \cap \widetilde{X})$, we obtain an isomorphism onto $Y \backslash\left(Y \cap X^{\prime \prime}\right)$.

Now recall that $q$ induces, by restriction, a projective line bundle $q: F \rightarrow X^{\prime \prime}$.

Since $X$ is general in $u_{n}$, we may also assume by Proposition 6(d) and Lemma 11 that $W:=\widetilde{\phi}(S)=q(\widetilde{S})$ is smooth at codimension 2 points and has, at worst, a finite number of double points of codimension 3; in particular, the singular set $\operatorname{Sing}(W)$ of $W$ may intersect $Y$ only for $n=5$ and does it, at worst, at a finite number of (closed) double points: indeed, since $X$ is general, we may suppose $S$ and $V$ to be generals containing $X^{\prime}$, and then $Y$ is the intersection of $W$ with a general hyperplane. Denote $U^{\prime \prime}:=X^{\prime \prime}-\left(X^{\prime \prime} \cap \operatorname{Sing}(W)\right)$.

On the other hand, the canonical exact sequence of vector bundles

$$
\left.0 \longrightarrow T_{U^{\prime \prime}} \longrightarrow T_{W}\right|_{U^{\prime \prime}} \longrightarrow N_{U^{\prime \prime}} W \longrightarrow 0
$$

associated to the normal line bundle $N_{U^{\prime \prime}} W$ of $U^{\prime \prime}$ in $W$, induces, after projectivization, a section of the projective line bundle $q: q^{-1}\left(U^{\prime \prime}\right) \rightarrow U^{\prime \prime}$. By construction, the closure of the image of this section is $\widetilde{S} \cap F$. Since $\widetilde{X}=\widetilde{q^{-1}}(Y) \subseteq \widetilde{S}$, we conclude that $\left.q\right|_{\widetilde{X} \cap F}: \widetilde{X} \cap F \rightarrow \widetilde{X^{\prime \prime}} \cap Y$ is an isomorphism if $n=4$ and contracts a fiber of $F$ onto each double point of $Y$ if $n=5$.

Combining this result with that of the first part of the proof, we obtain a birational morphism $\eta:=\left.q\right|_{\tilde{X}}: \widetilde{X} \rightarrow Y$ that is essentially the one we want; it remains to prove that, for $n=5$, the resolution $\eta$ is crepant.

First we observe that the Weil canonical divisor $K_{Y}$ of $Y$ is itself a Cartier divisor: since $Y$ is a hypersurface of degree 6 in $\mathbb{P}^{4}$, the divisor $K_{Y}$ is the closure of a hyperplane section $H_{Y}$; we can then move $H_{Y}$ and assume it lies in $Y \backslash \operatorname{Sing}(Y)$.

Finally, as $\eta$ induces an isomorphism from $\tilde{X} \backslash \eta^{-1}(Y \backslash \operatorname{Sing}(Y))$ onto $Y \backslash \operatorname{Sing}(Y)$ and $\eta^{-1}(\operatorname{Sing}(Y))$ is a reunion of a finite number of lines, we have $K_{\tilde{X}}=\eta^{*} K_{Y}$, completing the proof.

Remark 13. As shown in [Ein and Shepherd-Barron 1989, Proposition 2.3], the image by $p$ of the fibers $F_{x^{\prime \prime}}$ of $\left.q\right|_{F}: F \rightarrow X^{\prime \prime}$ for $x^{\prime \prime} \in X^{\prime \prime}$ are the $n$-secant lines of $X$. Then, for $n=5$, we eventually reach a finite number of $n$-secant lines of $X^{\prime}$ which are contained in $X$ and which will be contracted by $\phi$ to double points of $Y$. 
Therefore the existence of sectional singularities on $S$ depends on the existence of $n$-secant lines to $X^{\prime}$ that are contained in $X$ (compare with Remark 7(a)).

We have the following lemma:

Lemma 14. Let $m \geq 3$ be a positive integer. Let $Y_{1}, Y_{2} \subseteq \mathbb{P}^{m-1}$ be irreducible hypersurfaces of the same degree $r \geq m+1$ and $\varphi: Y_{1}-->Y_{2}$ a birational map. Assume that $Y_{1}$ and $Y_{2}$ are smooth if $m \leq 4$ and have at most isolated canonical singularities if $m \geq 5$. Then $\varphi$ is an isomorphism. If $r=m+1 \leq 5$ or $m \geq 5$, the morphism $\varphi$ extends to a linear automorphism of $\mathbb{P}^{m-1}$.

In the case of smooth hypersurfaces, the lemma above follows, as a particular case, from a theorem of Severi [1933], which it was generalized by C. Ciliberto [1987] for the case of Castelnouovo varieties. As we will see, we will use the lemma in a situation which is essentially covered by Ciliberto's theorem [Ciliberto 1987, Theorem 3.1.1]; however, for the convenience of the reader, we will give a complete proof, which leads with a slightly more general situation, though only for hypersurfaces.

Proof. The case $m=3$ is clear. Suppose that $m \geq 4$, and observe that the dualizing sheaf $\omega_{Y_{i}}^{0}$ of $Y_{i}$ is $\omega=\mathcal{O}_{Y_{i}}(\ell)=\mathcal{O}(\ell)$, where $\ell=r-m \geq 1$, for $i=1,2$.

Case 1: $m=4$. In this case, $Y_{i} \subseteq \mathbb{P}^{3}$ for $i=1,2$ is a smooth surface of general type. By Kodaira's vanishing theorem,

$$
\mathrm{H}^{1}\left(\omega^{\vee}\right)=\mathrm{H}^{1}\left(\omega^{\otimes 2}\right)=0,
$$

and it follows that $Y_{i}$ is minimal for $i=1,2$; see [Barth et al. 1984, Proposition VII.5.5]. Hence $\varphi$ is well defined; see [Bădescu 2001, Theorem 10.21].

Moreover, if $r=5$, the Riemann-Roch theorem implies

$$
h^{0}(\omega)-h^{1}(\mathcal{O})+h^{0}(\mathcal{O})=5 .
$$

From the exact sequence

$$
0 \longrightarrow \mathrm{O}_{\mathbb{P}^{3}}(-5) \longrightarrow \mathrm{O}_{\mathbb{P}^{3}} \longrightarrow \mathrm{O} \longrightarrow 0,
$$

we conclude that $h^{1}(\mathbb{O})=0$, and therefore $h^{0}(\omega)=h^{0}(\mathcal{O}(1))=4$. This means that an isomorphism $\varphi: Y_{1} \rightarrow Y_{2}$ comes from a linear isomorphism of $\mathbb{P}^{3}$.

Case 2: $m \geq 5$. Here $Y_{i} \subseteq \mathbb{P}^{4}$ for $i=1,2$ is an (m-2)-fold of degree $r \geq m+1$.

Let $H_{i}$ be a hyperplane section of $Y_{i}$ for $i=1,2$. By the Grothendieck-Lefschetz theorem (see for example [Hartshorne 1970, Corollary IV.3.2]), $\operatorname{Pic}\left(Y_{i}\right)=\mathbb{Z}\left[H_{i}\right]$, where $\left[H_{i}\right]$ is the hyperplane class of $Y_{i}$. Denote by $K_{i}$ a divisor associated to the dualizing sheaf $\omega_{Y_{i}}^{o}$ for $i=1,2$; then $K_{i}$ is linearly equivalent to $\ell H_{i}$. Observe that, by construction, $K_{i}$ is the Weil canonical divisor $K_{Y_{i}}$ of $Y_{i}$; then it is a Cartier divisor. 
On the other hand, take a resolution of indeterminancies of $\varphi$ that resolves also the singularities of $Y_{1}$, that is, a commutative diagram

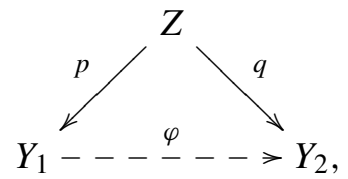

where $p$ and $q$ are birational morphisms and $Z$ is smooth. Take a canonical divisor $K_{Z}$ of $Z$, and take $H_{Z}=q^{*} H_{2}$. Since $Y_{i}$ has at most canonical singularities, we obtain $K_{Z}=q^{*} K_{2}+E_{q}=p^{*} K_{1}+E_{p}$, where $E_{q}$ and $E_{p}$ are effective divisors such that $q_{*}\left(E_{q}\right)=0$ and $p_{*}\left(E_{p}\right)=0$.

Now, we have $p_{*} q^{*}\left[H_{2}\right]=d\left[H_{1}\right]$ for a positive integer $d$; it is the class of the strict transform $H_{0}$ of $H_{2}$ by $\varphi^{-1}$. In particular, $H_{Z}=p^{*} H_{0}-F_{p}$, where $F_{p}$ is an effective divisor with $p_{*}\left(F_{p}\right)=0$.

Hence we have the numerically equivalent relations

$$
\begin{aligned}
K_{Z}-\ell H_{Z} \equiv q^{*}\left(K_{2}-\ell H_{2}\right)+E_{q} & \equiv E_{q} \\
& \equiv p^{*}\left(K_{1}-\ell H_{0}\right)+E_{p}+\ell F_{p} \\
& \equiv p^{*}\left((\ell-d \ell) H_{1}\right)+E_{p}+\ell F_{p},
\end{aligned}
$$

Applying $p_{*}$ we finally obtain that $(\ell-d \ell) H_{1}$ is numerically equivalent to an effective divisor, from which $d \leq 1$ and then $d=1$. Finally, since a general section of $Y_{i}$ is a smooth complete intersection, it is linearly normal; see [Hartshorne 1977, Example II.8.4]. Hence it is also true for $Y_{i}$, thus proving Lemma 14.

Proof of Theorem 9. For $n=3,4$ the theorem follows directly from Theorem 8 and Lemma 14. For $n=5$, the same results give a birational map $\varphi: X-->X$ which is an isomorphism in codimension 1 and which extends, by construction, to an automorphism of the crepant resolution $\eta: X \rightarrow Y$.

Proof of Theorem 10. The assertions "(a) implies (b)" and "(c) implies (b)" are trivial, and "(b) implies (c)" follows directly from Theorem 8 and Lemma 14 as in our proof of Theorem 9. Therefore it suffices to show that (b) implies (a).

There are Cremona transformations $\phi_{1}, \phi_{2}: \mathbb{P}^{n}--\rightarrow \mathbb{P}^{n}$ inducing, via restriction to $X_{1}$ and $X_{2}$, respectively, birational maps to hypersurfaces $Y_{1}, Y_{2} \subseteq \mathbb{P}^{n-1}$ (see Theorem 8); in particular, $Y_{1}$ and $Y_{2}$ are birationally equivalents.

Now, by Lemma 14, a birational map from $Y_{1}$ to $Y_{2}$ extends to a linear automorphism of $\mathbb{P}^{n-1}$; hence it extends to a linear automorphism of $\mathbb{P}^{n}$, which we denote by $\rho$. Thus $\phi_{2}^{-1} \circ \rho \circ \phi_{1}$ is a Cremona transformation mapping $X_{1}$ onto $X_{2}$ birationally. This completes the proof of Theorem 10 . 


\section{Acknowledgments}

I thank Igor Dolgachev for having asked me about the existence of a Cremona transformation of $\mathbb{P}^{3}$ transforming an ACM curve of degree 6 and genus 3 onto another one; that question initiated this paper.

I thank Francesco Russo, Ciro Ciliberto and Fernando Cuckierman for useful conversations.

I thank Claus Doering for his advice on the English of this paper.

\section{References}

[Arbarello et al. 1985] E. Arbarello, M. Cornalba, P. A. Griffiths, and J. Harris, Geometry of algebraic curves, I, Grundlehren der Mathematischen Wissenschaften 267, Springer, New York, 1985. MR 86h:14019 Zbl 0559.14017

[Bădescu 2001] L. Bădescu, Algebraic surfaces, Universitext, Springer, New York, 2001. MR 2001k:14068 Zbl 0965.14001

[Barth et al. 1984] W. Barth, C. Peters, and A. Van de Ven, Compact complex surfaces, vol. 4, Ergebnisse der Mathematik und ihrer Grenzgebiete (3), Springer, Berlin, 1984. MR 86c:32026 Zbl 0718.14023

[Cayley 1870] A. Cayley, "On the rational transformation between two spaces", Proc. London Math. Soc. 3 (1870), 127-180.

[Ciliberto 1987] C. Ciliberto, "On a property of Castelnuovo varieties", Trans. Amer. Math. Soc. 303:1 (1987), 201-210. MR 88e:14055 Zbl 0657.14022

[Crauder and Katz 1991] B. Crauder and S. Katz, "Cremona transformations and Hartshorne's conjecture”, Amer. J. Math. 113:2 (1991), 269-285. MR 92f:14011 Zbl 0754.14009

[Cremona 1871] L. Cremona, "Sulle transformazioni nello spazio", Lomb. Ist. Rend. 2:4 (1871), 269-279, 315-324.

[Debarre 2001] O. Debarre, Higher-dimensional algebraic geometry, Universitext, Springer, New York, 2001. MR 2002g:14001 Zbl 0978.14001

[Ein and Shepherd-Barron 1989] L. Ein and N. Shepherd-Barron, "Some special Cremona transformations”, Amer. J. Math. 111:5 (1989), 783-800. MR 90j:14015 Zbl 0708.14009

[Ellingsrud 1975] G. Ellingsrud, "Sur le schéma de Hilbert des variétés de codimension 2 dans $\mathbf{P}^{e}$ à cône de Cohen-Macaulay”, Ann. Sci. École Norm. Sup. (4) 8:4 (1975), 423-431. MR 52 \#13831 Zbl 0325.14002

[Fulton 1984] W. Fulton, Intersection theory, Ergebnisse der Mathematik und ihrer Grenzgebiete (3) 2, Springer, Berlin, 1984. MR 85k:14004 Zbl 0541.14005

[Gonzalez-Sprinberg and Pan 2006] G. Gonzalez-Sprinberg and I. Pan, "On characteristic classes of determinantal Cremona transformations", Math. Ann. 335:2 (2006), 479-487. MR 2007a:14021 Zbl 05035992

[Hartshorne 1970] R. Hartshorne, Ample subvarieties of algebraic varieties, Lecture Notes in Mathematics 156, Springer, Berlin, 1970. MR 44 \#211 Zbl 0208.48901

[Hartshorne 1977] R. Hartshorne, Algebraic geometry, Graduate Texts in Mathematics 52, Springer, New York, 1977. MR 57 \#3116 Zbl 0367.14001

[Hudson 1927] H. P. Hudson, Cremona Transformations in Plane and Space, Cambridge University Press, 1927. Zbl 53.0595.01 
[Kollár and Mori 1998] J. Kollár and S. Mori, Birational geometry of algebraic varieties, Cambridge Tracts in Mathematics 134, Cambridge University Press, 1998. MR 2000b:14018 Zbl 0926.14003

[Pan 1999] I. Pan, "Les applications rationnelles de $\mathbb{P}^{n}$ déterminantielles de degré $n$ ", An. Acad. Brasil. Ciênc. 71:3 (1999), 311-319. MR 2000h:14012 Zbl 0974.14036

[Pan and Russo 2005] I. Pan and F. Russo, "Cremona transformations and special double structures", Manuscripta Math. 117:4 (2005), 491-510. MR 2006f:14011 Zbl 02204884

[Peskine and Szpiro 1974] C. Peskine and L. Szpiro, "Liaison des variétés algébriques, I", Invent. Math. 26 (1974), 271-302. MR 51 \#526 Zbl 0298.14022

[Semple and Tyrrell 1970] J. G. Semple and J. A. Tyrrell, "The $T_{2,4}$ of $S_{6}$ defined by a rational surface ${ }^{3} F^{8}$,, Proc. London Math. Soc. (3) 20 (1970), 205-221. MR 41 \#5367 Zbl 0188.53404

[Severi 1933] F. Severi, "Sulla transformazione razionali di un'ipersuperficie algebrica priva di punti multiple", Math. Ann. 190 (1933), 4-6.

Received September 21, 2007. Revised March 5, 2008.

\section{IVAN PAN}

INSTITUTO DE MATEMÁTICA - UFRGS

Av. Bento GonÇAlves, 9500 - PrÉDio 43-111 - Agronomia

91509-900 PORTO ALEgre, RS

BRAZIL

pan@mat.ufrgs.br 